\title{
Continuing Medical Education
}

\section{Continuing medical education: Ultrasound guidance for regional blockade - basic concepts}

Ban C.H. Tsui MD frCPC, Derek Dillane MB MMedSci FFARCSI

ULTRASOUND GUIDANCE FOR REGIONAL BLOCKADE

Regional blockade has long afforded many benefits to the discipline of anesthesia. Despite this laudable record, it has been beleaguered by inconsistency. A reliance on blind techniques has made proficiency elusive and outcome unpredictable.

Now, for the first time in nearly 100 years of practice, ultrasound (US) technology provides regional anesthesiologists with the ability to visually locate the target nerve. The use of real-time US guidance in regional anesthesia is growing exponentially. Studies examining US imaging have demonstrated the superiority of US with respect to overall block success, ${ }^{1}$ reduced onset times, ${ }^{1}$ prolongation of block, ${ }^{2}$ and reduced complications. ${ }^{3,4}$

\section{Avoiding common mistakes when learning about and using US}

Although US imaging seems to have an infinite number of clinical benefits, visualization remains indirect to some extent because images are subject to individual perception and interpretation. The successful execution of a nerve block depends primarily on the operator's skill and technique. ${ }^{5}$ Some common mistakes in performing US guided regional blocks include: 1) failure to correctly localize the target structures due to inadequate scanning methodology; and 2) failure to ensure block success and safety by following the needle tip at all times. ${ }^{6}$ The objective of this continuing medical education (CME) module is to address the common mistakes made when learning about and using US technology. In addition, the module high- lights a number of useful maneuvers in an attempt to avoid common pitfalls which may ultimately shorten the extensive learning curve associated with this technique.

\section{Scanning technique for structure identification} The clinician should neither expect nor seek to locate the target nerve at first placement of the US probe. A structured scan at each block site using consistent and recognizable anatomical prompts will yield greater results. It has been stated that confidence, experience, and knowledge all influence the success of anesthetic placement. ${ }^{7}$ Ultrasound-guidance can positively influence knowledge and confidence, but the technique does not eliminate the need for experience. Experience using US for structure identification can be gained by practising imaging and needling techniques on cadavers, in gel or on animal phantoms. ${ }^{8}$ Ultimately, knowledge of the regional anatomy, coupled with a command of the US system being used, can be vital to successful block execution.

\section{Knowledge of regional anatomy}

Knowledge of regional anatomy is of fundamental importance before attempting to interpret perceived sonoanatomy. Some US images can appear nebulous to the uninitiated, and a command of anatomy will reduce early frustration. The distinct echotexture of the nerves and their surrounding structures are visualized through their reflection of US waves emitted by a transducer. Size, shape, and echogenicity help to distinguish the nerves from surrounding tissues. ${ }^{9}$ Nearby bony structures and blood vessels are often used for verification

From the Department of Anesthesiology and Pain Medicine, University of Alberta, Edmonton, Alberta, Canada.

Address correspondence to: Dr. Ban C.H. Tsui, Department of Anesthesiology and Pain Medicine, University of Alberta Hospitals, 8-120 Clinical Sciences Building, Edmonton, Alberta T6G 2G3, Canada. Phone: 780-407-8861; Fax: 780-407-3200; E-mail: btsui@ualberta.ca; www.EdmARA.ca

Supported, in part, by an Education and Research Fund, Department of Anesthesiology and Pain Medicine, University of Alberta Hospitals, Edmonton, Canada and by a Clinical Investigatorship Award, Alberta Heritage Foundation for Medical Research, Alberta, Canada. 
TABLE Useful ultrasound landmarks to aid in the identification of nerves while performing an ultrasound-guided regional blockade

\begin{tabular}{ll}
\hline Block & Useful ultrasound landmark \\
\hline $\begin{array}{l}\text { Brachial plexus } \\
\text { Interscalene }\end{array}$ & $\begin{array}{l}\text { Subclavian artery* } \\
\text { Supraclavicular }\end{array}$ \\
$\begin{array}{l}\text { Subclavian artery } \\
\text { Infraclavicular }\end{array}$ & $\begin{array}{l}\text { Subclavian artery \& vein } \\
\text { Axillary }\end{array}$ \\
$\begin{array}{l}\text { Lumbary artery } \\
\text { Peripheral nerves }\end{array}$ & Transverse processes \\
Median at antecubital fossa & Brachial artery \\
Radial at anterior elbow & Humerus at spiral groove* \\
Ulnar at medial forearm & Ulnar artery \\
Femoral & Femoral artery \\
Sciatic (labat) & Ischial bone \\
Sciatic (subgluteal) & Greater trochanter and ischial \\
& tuberosity \\
Sciatic (popliteal) & Popliteal artery* \\
Tibial (posterior tibial) at ankle & Posterior tibial artery \\
Deep peroneal (at ankle) & Anterior tibial artery \\
\hline
\end{tabular}

*Indicates that the ultrasound "traceback" approach may be useful in locating the nerve."

Table adapted from: Tsui BCH. Atlas of Ultrasound and Nerve Stimulation-Guided Regional Anesthesia. New York: Springer Science+Business Media LLC; 2007.

of nerve identity and can be used as anatomical landmarks. ${ }^{10}$ It is very useful to locate these anatomical landmarks first, as they are highly identifiable under US (Table). With the US probe in the transverse plane, the "traceback" technique can be used over short distances to distinguish and identify various anatomic structures. ${ }^{11}$ This scanning technique can also be used to find a more desirable block location (Figure 1 ; video online) ${ }^{12}$

Previous studies have shown that the US images obtained from a cadaver are similar to those of a live subject. ${ }^{12-14}$ Utilizing cadavers for ultrasonic anatomical studies and nerve localization may provide the knowledge and ability to successfully identify the nerve and its surrounding structures. Once regional anatomy is understood, the capabilities and limitations of the US machine must be appreciated before needling is attempted.

\section{Knowledge of US machine BEAM DIRECTION}

One of the major obstacles in successfully administering a US-guided regional block is the inability to comprehend probe placement and transducer configuration. The US transducer serves as both a transmitter and a receiver of US waves. These waves are subject to reflection, refraction, and scattering on impact with the target. Attainment of a clear target image is dependent on the return of a strong signal to the

\section{Posterior Thigh}

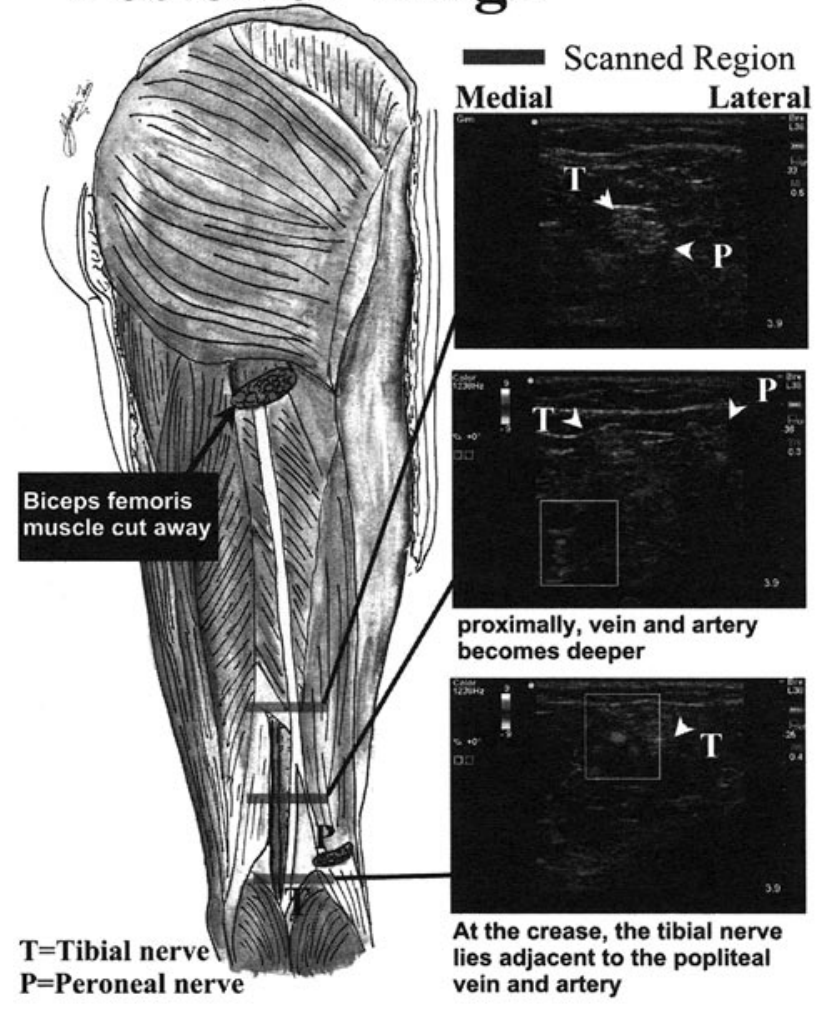

FIGURE 1 Traceback approach for popliteal sciatic nerve.

transducer. ${ }^{3}$ It is important to understand that the US beam provides optimal imaging when the waves are perpendicular to the axis of the target structure since refraction is minimized and reflection is maximized. ${ }^{15}$ Image distortion may result if this so-called angle of incidence is substantially different.

\section{SHAPE OF TRANSDUCER}

When using US, image clarity and successful structure identification can be significantly affected by the selection of an appropriate transducer. Linear and convex transducers are the most commonly used probes for reglonal anesthesia. Linear transducers create a rectangular image and produce the same uniform picture in fields both near and far. ${ }^{16}$ It is frequently used for small areas and can be used when the nerve structures are superficially situated in the tissue. ${ }^{17}$ Convex transducers, also referred to as curved probes, yield deeper penetration and produce a larger wedge-shaped image with less coupling to the contact area. ${ }^{18}$ This larger image field affords a more complete view of the needle path during its trajectory, thus increasing block safety. ${ }^{19}$ The primary disadvantage of the convex trans- 


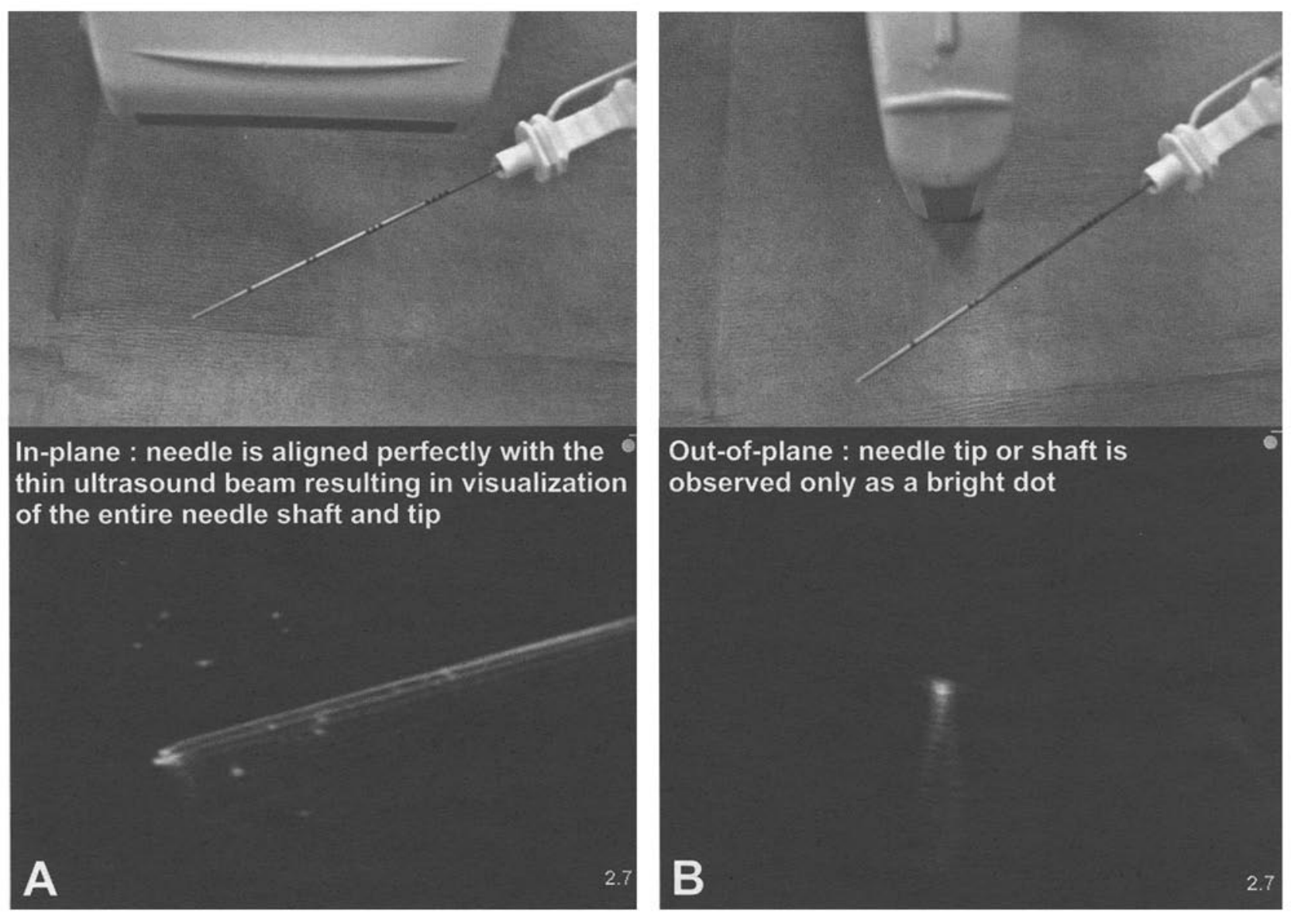

FIGURE 2 Needling using in-plane and out-of-plane approaches.

ducer is that it produces a curved image that may be difficult for a beginner to interpret. High frequency probes allow high resolution but have limited depth of penetration; lower frequency probes allow greater depth but with less resolution. Consequently, low frequency probes are suitable for neuraxial, lumbar plexus, and trans-gluteal blocks.

\section{ARTIFACTS}

Even the most sophisticated US equipment is limited by artifact generation. An artifact is any perceived distortion, error, or addition to the image that is not truly representative of the area being scanned. ${ }^{15}$ The four categories of imaging artifacts are as follows: 1 ) acoustic; 2) anatomic; 3) error in perception/optical illusion; or 4) electrical noise. ${ }^{20}$ Acoustic and anatomic are the most commonly encountered artifacts in regional anesthesia. ${ }^{15}$ Acoustic artifacts are often a consequence of user interface issues, such as inappropriate use of gain, depth, and frequency controls. ${ }^{18}$ They usually result in either missing or falsely perceived structures and degraded images. ${ }^{15}$ Anatomic artifacts are a misinterpretation of an actual tissue structure (e.g., tendons and muscles) that may resemble the target nerve. ${ }^{9,15}$ For nerve confirmation and for tracing the target nerve along its anatomical pathway, the errors associated with artifacts can be minimized by using nerve stimulation in conjunction with US.,15

Failure to become familiar with the relevant regional anatomy and operational abilities of the US machine will greatly diminish the ability of the anesthesiologist to identify the appropriate structures for successful nerve blockade.

\section{Procedural skills for needling}

The one outstanding attribute of US-guided nerve blockade is the ability to dynamically adjust and finetune local anesthetic deposition around the target nerve while simultaneously avoiding nearby structures. Ultrasound imaging provides real-time visualization, thus giving the operator the ability to adjust needle placement instantaneously. ${ }^{21}$ When examining 
the learning curve associated with US-guidance, Sites et $a .^{22}$ found that anesthesia residents mastered most basic US skills quite rapidly; however, $70 \%$ of the residents failed to accurately locate the needle while advancing. This finding demonstrates that knowledge of basic needle-placement techniques are a prerequisite for any regional anesthesiologist attempting USguided blockade. ${ }^{22}$

\section{In-plane needle alignment}

In-plane (longitudinal, long-axis) needle alignment refers to aligning the needle with the long-axis of the probe so that the entire shaft and tip of the needle is visible (Figure 2a). ${ }^{23}$ One of the disadvantages of the in-plane needle view, as a result of the US beam being thin, is the ease with which the image can be lost with a slight movement of the probe. ${ }^{24}$ When using the longitudinal approach, the needle can be inserted either by means of a needle-guide apparatus or by "free-hand" ${ }^{24}$ The freehand technique is preferable, as it permits tracking of the needle trajectory in real time allowing fine adjustments to needle placement, and it does not require expensive needle-guide apparatus. ${ }^{25}$ However, this technique requires excellent hand-eye coordination and may require a longer learning curve ${ }^{25}$ Although further studies are required, a portable laser unit can improve needle visibility and can aid in teaching proper in-plane alignment. ${ }^{25}$ Such devices may successfully shorten the individual learning curve associated with US-guided blocks and needle placement.

\section{Out-of-plane alignment}

The out-of-plane (i.e., transversal, tangential, shortaxis) needle approach results in the needle crossing the plane of imaging near the target. ${ }^{11}$ Since only the needle tip is observed as a bright dot, it is sometimes difficult to accurately observe the needle during advancement (Figure 2b). ${ }^{18,26}$ Despite this, it has been shown that novice US users were able to obtain vascular access more quickly when using an out-of-plane approach than when using an in-plane alignment. ${ }^{27}$ When using the out-of-plane approach, there are two techniques that can be used to aid in correct needle placement, i.e., the small-volume injection test ${ }^{24}$ and the "walk-down" method. ${ }^{26}$ The small-volume injection test enhances the view of the needle tip location by injecting a small amount of $5 \%$ dextrose in water. Air bubbles and local anesthetic solution should not be injected, as this will result in acoustic shadowing, thus hindering nerve stimulation. ${ }^{23,28}$ The "walkdown" approach uses the US to determine the distance/depth of the target nerve. The puncture site is

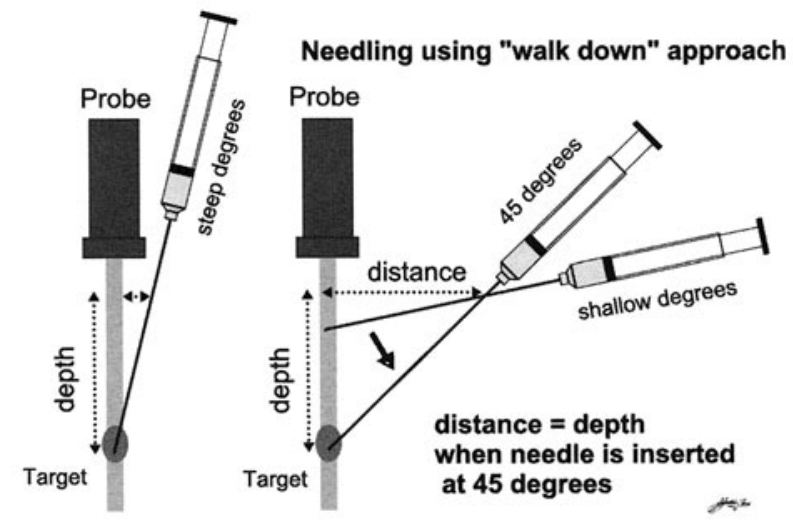

FIGURE 3 Out-of-plane needling using "walk down" technique.

of equal distance from the probe as the target depth. The needle is then inserted at a shallow angle and tracked at progressively steeper angles until the target nerve is reached at approximately $45^{\circ}$ (Figure 3 ). ${ }^{26}$ Both techniques will enable improved visibility of the needle tip while decreasing the chance of possible errors.

\section{Technique combination: nerve stimulation and US}

As laudable as it is, US technology does not abolish uncertainty with regard to nerve identification. Surrogate visualization of the needle and nerve as well as a reasonable estimate of the spread of local anesthetic may usually be determined, yet the correct identity of the nerve may remain questionable. Nerve stimulation is a common modality used in regional anesthesia to identify nerve structures based on their response to an electrical current. ${ }^{29}$ By electrically stimulating that target structure, the clinician can objectively determine its identity by observing the distinct motor response to nerve stimulation. ${ }^{4}$ However, due to the interindividual variation often seen in response to stimulation, this diagnostic device is not $100 \%$ effective in identifying the target nerve. ${ }^{30-32}$ Both nerve localization techniques have their respective limitations; however, in combination they may compensate for each other's weaknesses to further approach the goal of a $100 \%$ success rate and possibly shorten the learning curve associated with each approach. ${ }^{33}$

\section{Conclusion}

The practice of regional anesthesia has long been regarded as an "art" confined to the resolute enthusiast. Ultrasound guidance is now at the vanguard of educational development in this discipline and may help to transform it into a "science" accessible to all. 
It is important to keep in mind, however, that US waves alone do not induce nerve blockade; they merely facilitate it.

In summary, to obtain complete competence and decrease the incidence of errors made in US imaging, successful training in both cognitive and procedural abilities is required ${ }^{34}$ We must strive to obtain a $100 \%$ block success rate and, with the successful use of US, we may eventually reach that target.

\section{Ultrasound module objectives}

1) To address the common mistakes made when learning about, and using, ultrasound-guided peripheral regional anesthesia.

2) To discuss how to successfully locate a target nerve structure using ultrasound.

3) To describe and compare the various needle approaches used in ultrasound-guided anesthesia.

4) To limit errors by incorporating the use of two commonly used modalities in anesthesia into one approach, i.e., ultrasound and nerve stimulation.

\section{Instructions for completing the CME module}

1) Read the references indicated in bold.

2) Visit the Canadian Journal of Anesthesia website (www.cja-jca.org), click "CME online", and select the current module (Ultrasound Guidance for Regional Blocks).

3) Answer the multiple choice questions regarding a case (briefly outlined below).

4) After entering all of your answers, you will have access to an expert's explanation for all of the possible choices.

5) Participants may claim up to ten hours, for a total of 20 credits, under section 3 of the Continuing Professional Development (CPD) program of the Royal College of Physicians and Surgeons of Canada.

\section{The Case and questions}

A 68-yr-old male presents for repair of an Achilles tendon rupture that is to be performed under a sciatic nerve block at the popliteal fossa. Ultrasound guidance and nerve stimulation are used to aid block placement. The needle is advanced out-of-plane, and ankle dorsiflexion is noted. Tissue distortion is discernible, but the needle is not clearly seen.

1. How do you proceed with nerve localization?

2. How should the ultrasound equipment be used?

3. What is true about imaging artifacts?

4. What should be avoided when trying needle alignment?

5. Is nerve stimulation useful with ultrasound?
The block is deemed to be successful, and the patient is ready for surgery after $25 \mathrm{~min}$. The surgeon informs you that he wishes to use a calf tourniquet.

6. What is the best course of action?

7. When performing peripheral blocks, knowledge of the relevant anatomy is as important when using ultrasound guidance as it is when using conventional approaches. Correlation of neural structures with surrounding tissue and identification of individual nerves is paramount. The participant should identify the tibial nerve on an image that will be presented.

8. The participant should identify the femoral nerve on an ultrasound image taken at the inguinal crease in transverse view with medial and lateral orientation shown.

\section{References}

1 Chan VW, Perlas A, McCartney CJ, Brull R, Xu D, $A b b a s S$. Ultrasound guidance improves success rate of axillary brachial plexus block. Can J Anesth 2007; 54: 176-82.

2 Marbofer P, Sitzwohl C, Greher M, Kapral S. Ultrasound guidance for infraclavicular brachial plexus anaesthesia in children. Anaesthesia 2004; 59: 642-6.

3 Marhofer $P$, Chan VW. Ultrasound-guided regional anesthesia: current concepts and future trends. Anesth Analg 2007; 104: 1265-9.

4 Tsui B. Ultrasound-guidance and nerve stimulation: implications for the future practice of regional anesthesia. Can J Anesth 2007; 54: 165-70.

5 Marhofer $P$, Greher $M$, Kapral S. Ultrasound guidance in regional anaesthesia. Br J Anaesth 2005; 94: 7-17.

6 Sites BD, Spence BC, Gallagher JD, Wiley $C W$, Bertrand $M L$, Blike GT. Characterizing novice behavior associated with learning ultrasound-guided peripheral regional anesthesia. Reg Anesth Pain Med 2007; 32: 107-15.

7 Grau T, Bartusseck E, Conradi R, Martin E, Motsch $J$. Ultrasound imaging improves learning curves in obstetric epidural anesthesia: a preliminary study. Can J Anesth 2003; 50: 1047-50.

8 Broking $K$, Waurick $R$. How to teach regional anesthesia. Curr Opin Anaesthesiol 2006; 19: 526-30.

9 Silvestri E, Martinoli C, Derchi LE, Bertolotto M, Chiaramondia $M$, Rosenberg I. Echotexture of peripheral nerves: correlation between US and histologic findings and criteria to differentiate tendons. Radiology 1995; 197: 291-6.

$10 T$ sui $B C$. Innovative approaches to neuraxial blockade in children: the introduction of epidural nerve root stimulation and ultrasound guidance for epidural catheter placement. Pain Res Manag 2006; 11: 173-80. 
11 Tsui BC, Finucane BT. The importance of ultrasound landmarks: a "traceback" approach using the popliteal blood vessels for identification of the sciatic nerve. Reg Anesth Pain Med 2006; 31: 481-2.

12 Tsui BC, Dillane D, Walji $A H$. Cadaveric ultrasound imaging for training in ultrasound-guided peripheral nerve blocks: upper extremity. Can J Anesth 2007; 54: 392-6.

13 Tsui BC, Dillane D, Pillay J, Ramji AK, Walji $A H$. Cadaveric ultrasound imaging for training in ultrasound-guided peripheral nerve blocks: lower extremity. Can J Anesth 2007; 54: 475-80.

14 Tsui B, Dillane D, Pillay J, Walji A. Ultrasound imaging in cadavers: training in imaging for regional blockade at the trunk. Can J Anesth 2008; 55: 105-11.

15 Sites $B D$, Brull $R$, Chan VW, et al. Artifacts and pitfall errors associated with ultrasound-guided regional anesthesia. Part II: a pictorial approach to understanding and avoidance. Reg Anesth Pain Med 2007; 32: 419-33.

16 Marhofer $P$, Frickey $N$. Ultrasonographic guidance in pediatric regional anesthesia part 1 ; theoretical background. Paediatr Anaesth 2006; 16: 1008-18.

17 Tsui $B$. Equipment for regional anesthesia in children. Tech Reg Anesth Pain Man 2007; 11 : 235-46.

18 Sites BD, Spence BC, Gallagher J, Beach ML, Antonakakis JG, Sites VR, Hartman GS. Regional anesthesia meets ultrasound: a specialty in transition. Acta Anaesthesiol Scand 2008; 52: 456-66.

19 Tsui BC, Ozelsel TJ. Ultrasound-guided anterior sciatic nerve block using a longitudinal approach: "expanding the view”. Reg Anesth Pain Med 2008; 33: 275-6.

20 Kremkau FW, Taylor KJ. Artifacts in ultrasound imaging. J Ultrasound Med 1986; 5: 227-37.

21 Peterson $M K$, Millar FA, Sheppard DG. Ultrasoundguided nerve blocks. Br J Anaesth 2002; 88: 621-4.

22 Sites BD, Gallagher JD, Cravero J, Lundberg J, Blike $G$. The learning curve associated with a simulated ultrasound-guided interventional task by inexperienced anesthesia residents. Reg Anesth Pain Med 2004; 29: 544-8.

23 Gray AT. Ultrasound-guided regional anesthesia: current state of the art. Anesthesiology 2006; 104: 368-73.

24 Chapman GA, Johnson D, Bodenham AR. Visualisation of needle position using ultrasonography. Anaesthesia $2006 ; 61: 148-58$.

$25 T$ sui $B C$. Facilitating needle alignment in-plane to an ultrasound beam using a portable laser unit. Reg Anesth Pain Med 2007; 32: 84-8.

26 Tsui $B C$, Dillane $D$. Needle puncture site and a "walkdown" approach for short-axis alignment during ultrasound-guided blocks. Reg Anesth Pain Med 2006; 31: 586-7.

27 Blaivas $M$, Brannam L, Fernandez E. Short-axis versus long-axis approaches for teaching ultrasound-guided vascular access on a new inanimate model. Acad Emerg Med 2003; 10: 1307-11.

28 Tsui BC, Kropelin B. The electrophysiological effect of dextrose $5 \%$ in water on single-shot peripheral nerve stimulation. Anesth Analg 2005; 100: 1837-9.

29 Hudes E, Lee KC. Clinical use of peripheral nerve stimulators in anaesthesia. Can J Anaesth 1987; 34: 525-34.

30 Sauter AR, Dodgson MS, Stubhaug A, Cvancarova $M$, Klaastad $O$. Ultrasound controlled nerve stimulation in the elbow region: high currents and short distances needed to obtain motor responses. Acta Anaesthesiol Scand 2007; 51: 942-8.

31 Beach $M L$, Sites BD, Gallagher JD. Use of a nerve stimulator does not improve the efficacy of ultrasoundguided supraclavicular nerve blocks. J Clin Anesth 2006; 18: 580-4.

32 Choyce A, Chan VW, Middleton WJ, Knight PR, Peng $P, M c$ Cartney CJ. What is the relationship between paresthesia and nerve stimulation for axillary brachial plexus block? Reg Anesth Pain Med 2001; 26: 100-4.

33 Orebaugh SL, Williams BA, Kentor ML. Ultrasound guidance with nerve stimulation reduces the time necessary for resident peripheral nerve blockade. Reg Anesth Pain Med 2007; 32: 448-54.

34 de Oliveira Filho GR, Helayel PE, da Conceicao DB, Garzel IS, Pavei P, Ceccon MS. Learning curves and mathematical models for interventional ultrasound basic skills. Anesth Analg 2008; 106: 568-73. 